\title{
Fortaleciendo el Clima Moral en escuelas de educación básica en Ciudad Juárez
}

\author{
Oliverio Ismael Ferman Ávila \\ Subsecretaría Educación, Cultura y Deporte en la Zona Norte \\ Programa Educación en Valores \\ ismael.ferman@educacionyvaloresj.org
}

\section{Resumen}

Este artículo pretende aportar elementos conceptuales e instrumentales de la educación en valores, a partir de un esfuerzo de investigación-evaluación de los impactos del proyecto de intervención del Programa Educación en Valores (PEV). En su planteamiento, el PEV se enfoca en el concepto de clima moral, concibiendo a la escuela como el espacio donde se concretan un conjunto de acciones a través de acuerdos sociales y prácticas sistemáticas aunque no siempre realizadas de manera intencional, pero siempre supeditadas a valores y/o actitudes. El PEV pretende influir en la generación de valores morales o éticos, por medio de un planteamiento que se orienta a intervenir en el clima organizacional de la escuela, mediante en dos referentes: El clima moral y las relaciones interpersonales. El PEV ha venido considerando estos dos referentes como las unidades de análisis centrales por medio de las cuales se pretende determinar el avance de los procesos educativos que estimulan acuerdos sociales o valores entre directivos, profesores, estudiantes y padres de familia. El PEV se propone desde dos marcos de investigación: La reconstrucción de valores a través de la orientación transversal de la gestión del currículo; y la construcción de valores y desarrollo de actitudes a través del propio proyecto institucional de gestión escolar. Para ello el PEV realiza una asesoría al colectivo escolar en la elaboración de una propuesta para cada escuela en particular, que permita a la comunidad escolar afianzar valores y acuerdos sociales orientados al desarrollo académico y promover la convivencia armónica dentro de la comunidad escolar (Ferman, 2008).

\section{Palabras clave}

Educación en valores, clima escolar, comunicación educativa.

\section{Introducción}

El Programa Educación en Valores (PEV), busca por medio de una evaluación de impacto identificar factores contextuales al interior de la escuela, que puedan estar determinando el desarrollo de la conciencia ética y de la ciudadanía de los estudiantes, buscando incidir con ello en el clima moral de la escuela.

Consistentemente la evaluación que se ha venido planteando en el PEV se centra en el conocimiento de percepciones de directivos, profesores, padres de familia y alumnos; elementos donde se ha venido verificando el impacto de su proyecto de intervención que actualmente se desarrolla en 635 escuelas de educación básica en Ciudad Juárez.

Este trabajo busca dar a conocer algunos elementos indicativos más allá de 
las percepciones, aspectos específicos en los cuales la propuesta de educación en valores del PEV se encuentra impactando, y que resultan indispensables en la propia idea del proyecto. Dos elementos indicativos a este respecto los constituyen la Ideología Educativa y el Razonamiento Moral (Anaya, 2012). Como complemento y en atención a la importancia del clima moral, se incluye el Liderazgo en los Centros Docentes; el cual permite realizar una aproximación incluyente y más concreta al estilo de liderazgo ejercido por los directivos, e identificar la existencia de una sinergia con relación al clima escolar percibido por los diferentes participantes del colectivo escolar.

La pregunta central que orientó este estudio es: ¿En qué medida el proyecto de valores éticos (PROVEE) que desarrolla el Programa Educación en Valores en escuelas del nivel básico en Cd Juárez, Chihuahua contribuye a Mejorar el clima moral de la institución mediante el diseño y/o implementación de un Proyecto de Valores Éticos (PROVEE), y de esta manera permitir que a largo plazo se impacte en la calidad educativa?

En el levantamiento de los datos se establecen a partir de la variable dependiente Clima Moral. Este tiene como referencia los valores inmersos en el clima moral según la propuesta del PEV.

En la propuesta de clima moral se distinguen dos aspectos interrelacionados: a) Propuesta axiológica (los valores) y las variables (aspectos observables en la realidad).

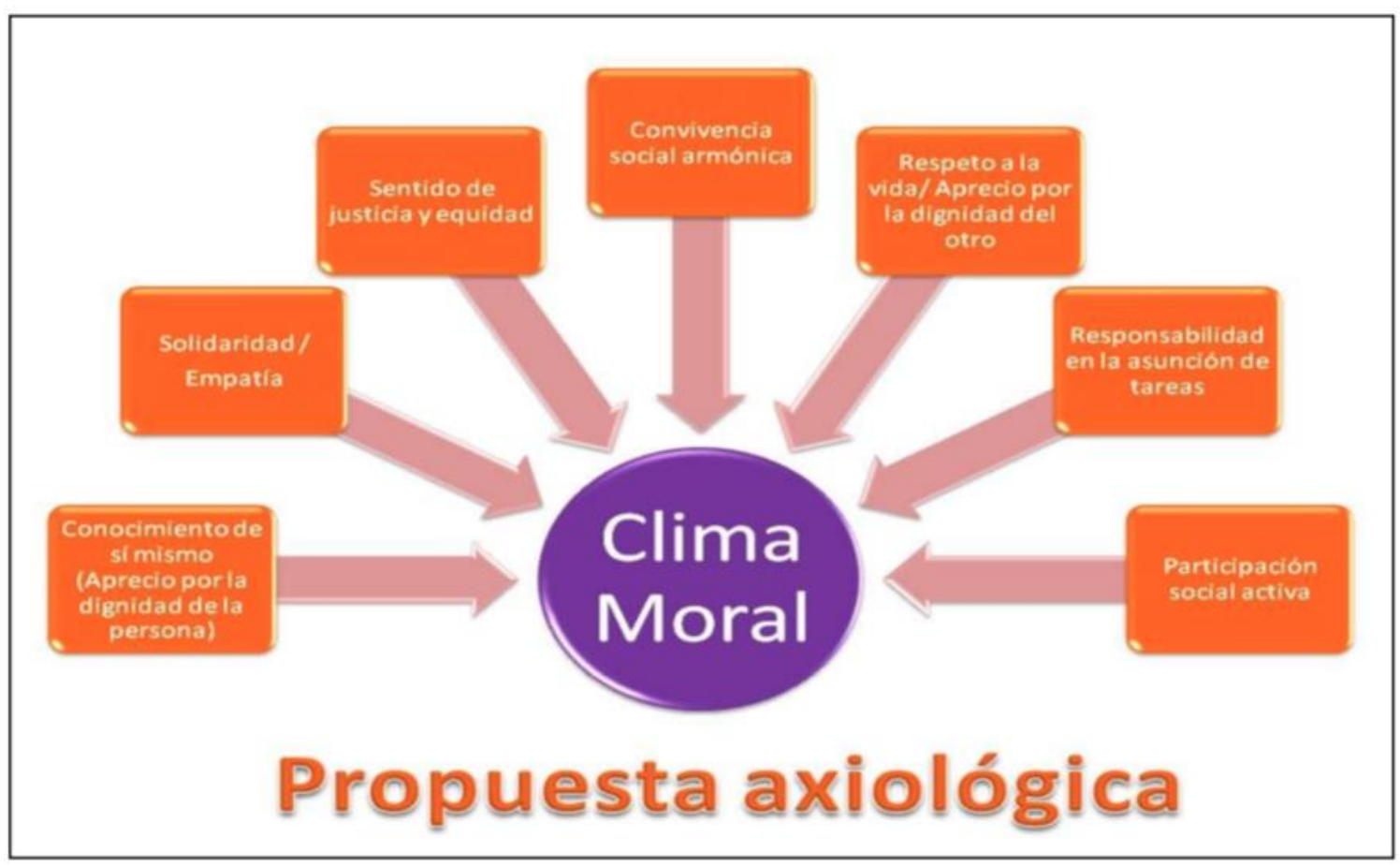

Ferman Ávila, O.I. 


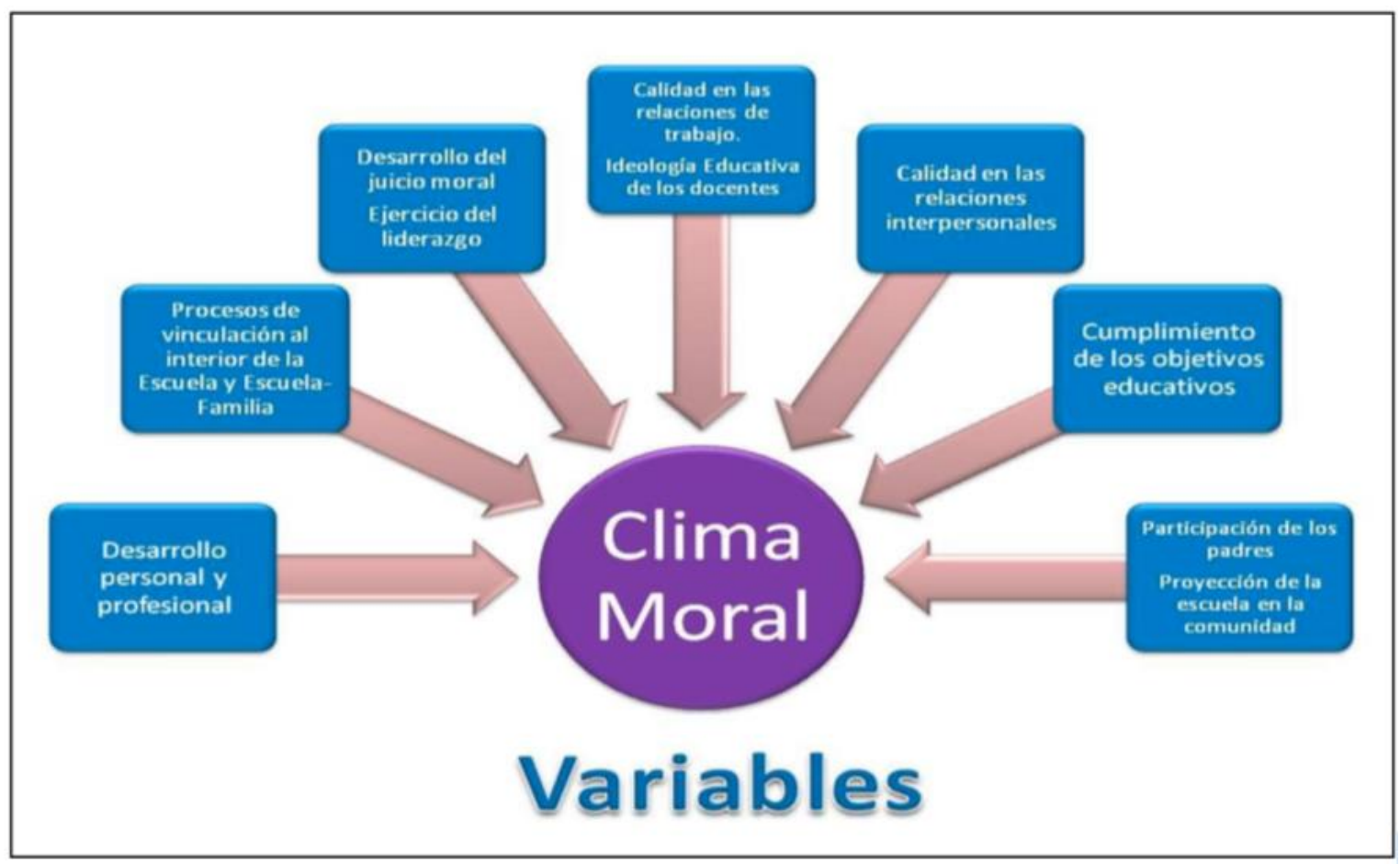

A continuación, se establecen los términos centrales que orientaron el desarrollo de la investigación:

a) Proyecto de Valores Éticos en la Escuela (PROVEE). Es el proyecto que asesora el Programa Educación en Valores en las escuelas de educación básica en Ciudad Juárez para complementar al proyecto académico y lograr posibilitar un proyecto educativo integral de calidad. Este es considerado el proyecto de intervención del PEV en las escuelas de educación básica de ciudad Juárez.

b) Proyecto Escolar. Se trata de una propuesta integral que contextualizada en la realidad concreta y definiendo las propias metas de identidad, permite llevar a cabo, de forma coherente y eficaz, los procesos educativos de la escuela.
a. Asimismo, es un instrumento que posibilita

encontrar justificación o respuesta a las decisiones que se toman, tanto en el Proyecto Curricular y los Programas Específicos de las Âreas, como las relativas a la organización y gestión.

b. Su función básica es la de proporcionar un marco global de referencia a la institución escolar, que permita la actuación coordinada y eficaz del equipo docente y de toda la comunidad educativa general.

c) Clima escolar. Se valora por la calidad, o bien por la satisfacción, de las relaciones entre sus miembros y los sentimientos de aceptación y de rechazo de los demás. Un buen clima escolar induce a una convivencia más fácil y permite abordar los conflictos en mejores condiciones. Es un factor 
que incide en la calidad de la enseñanza que imparte. Según la propuesta del PEV en el clima moral se encuentran inmersos cuando menos 6 valores: Conocimiento de sí (aprecio por la dignidad propia) - desarrollo personal-; Solidaridad/ empatía; Sentido de justicia y equidad; Convivencia social armónica; Respeto a la vida y aprecio por la dignidad del otro; Responsabilidad en la asunción de tareas; y, Participación social activa.

d) Razonamiento Moral. Grado de desarrollo- (Kohlberg, 1992). Se considera un elemento significativo en el desarrollo de las cualidades cognitivas, con un alcance mayor al mero razonamiento de lógica formal; además integra una categoría ética del comportamiento. La teoría del desarrollo moral se circunscribe en las tesis fundamentales de Piaget sobre el desarrollo (Reimer y otros, 1983), las cuales plantean que el desarrollo ocurre porque actuamos como intérpretes activos de nuestra experiencia y entonces construimos significados para entenderla. Para ello nos hacemos categorías generales de significados, en donde las experiencias se asimilan y se forman expectativas de posibles consecuencias. Sin embargo, al presentarse nuevas experiencias que no pueden asimilarse en las categorías previas, procedemos a revisar dichas categorías, con lo que se emprende el cambio cognitivo, así el desequilibrio cognitivo es condición para el desarrollo. e) Ideología Educativa. (Wiliam O’Neill -1981). Comprende el conjunto de opiniones, actitudes y valores acerca de la educación, que tienen los maestros y las maestras. Que implica la perspectiva acerca del propósito de la educación, los objetivos secundarios, el posicionamiento del alumno en el proceso de enseñanza aprendizaje, la selección del conocimiento válido, la importancia del control disciplinario, la función social y profesional del maestro, los valores deseables a promover, la concepción de la escuela como organización institucional y el diseño curricular de las experiencias educativas que implica la participación de la comunidad en general, y de manera particular, de los padres de familia y del Estado (Loera, 2005; Arroyo, 1998: 67; O’Neill, 1981).

f) Liderazgo educativo en el centro. El liderazgo ejercido por los directivos en los centros docentes, que distinga entre liderazgos meramente transaccionales, transformacionales o no liderazgos (Pascual, Villa y Auzmendi, 1993), ofrecerá elementos indicadores de las dinámicas bajo las cuales se configurarán e implementarán los proyectos y programas planteados dentro del PEV. Igualmente, permite conocer la manera en que los profesores identifican el liderazgo ejercido por el directivo, para posteriormente realizar los consecuentes contrastes, tanto al interior de la escuela como entre escuelas.

g) ENLACE (Evaluación Nacional del Logro Académico en Centros 
Escolares). La prueba ENLACE es un instrumento que constituye una serie de procesos tanto de evaluación de resultados como de calidad educativa y por lo tanto, la transparencia de los procedimientos que se llevan a cabo en estos procesos, deben ser custodiados por la sociedad para su efectividad y cumplimiento de objetivos.
Levantamiento de datos para la evaluación

La finalidad del presente estudio fue la de recabar información evaluativa, tanto cuantitativa como cualitativa, en los miembros de las comunidades escolares donde se implementa el Proyecto de Valores Éticos en las Escuelas (PROVEE), en sus diferentes ciclos de implementación. En el siguiente cuadro se especificas muestras que se consideraron para el levantamiento de información cuantitativa:

Cuadro 1. Muestras, criterios de selección y diseño de investigación.

\begin{tabular}{|c|c|c|c|}
\hline MUESTRA A & \multicolumn{1}{|c|}{$\begin{array}{c}\text { CRITERIO DE } \\
\text { SELECCIÓN }\end{array}$} & $\begin{array}{c}\text { Diseño de } \\
\text { Investigación }\end{array}$ & $\begin{array}{c}\text { Muestra } \\
\text { \# } \\
\text { Escuelas) }\end{array}$ \\
\hline \multirow{4}{*}{ A } & $\begin{array}{l}\text { 1. Escuelas que se ubican la } \\
\text { Fase V del primer ciclo de } \\
\text { implementación del PROVEE. }\end{array}$ & $\begin{array}{c}\text { Diseño no experimental } \\
\text { longitudinal de } \\
\text { tendencia (trend). }\end{array}$ & 57 \\
\cline { 2 - 4 } & $\begin{array}{l}\text { 2. Escuelas que se ubican en las } \\
\text { Fases I del primer ciclo de } \\
\text { implementación del PROVE. } \\
\text { Escuelas testigo) -muestra no } \\
\text { probalística-. }\end{array}$ & $\begin{array}{c}\text { Diseño no experimental } \\
\text { longitudinal de } \\
\text { tendencia (trend). }\end{array}$ & 20 \\
\hline B & $\begin{array}{l}\text { Escuelas que se ubican en la } \\
\text { Fase V del segundo ciclo de } \\
\text { implementación del PROVEE. }\end{array}$ & $\begin{array}{c}\text { Diseño longitudinal de } \\
\text { panel }\end{array}$ & 60 \\
\hline C & $\begin{array}{l}\text { Escuelas que se ubican en la } \\
\text { Fase V del tercer ciclo de } \\
\text { implementación del PROVEE. }\end{array}$ & $\begin{array}{c}\text { Diseño longitudinal de } \\
\text { panel }\end{array}$ & 6 \\
\hline
\end{tabular}

Para el levantamiento de información suficiente, tanto cuantitativa como cualitativa, se determinaron diversas técnicas de recolección de información, que se especifican en el siguiente cuadro: 
Cuadro 2. Técnicas de recolección de datos

\begin{tabular}{|c|c|}
\hline \multicolumn{2}{|c|}{ Técnicas de Recolección de datos } \\
\hline Cualitativas & Cuantitativas \\
\hline - Entrevistas a profundidad. & $\begin{array}{c}\text { moral. } \\
\text { - Grupo focal. }\end{array}$ \\
& - Prueba de definición de Criterios de \\
James Rest (Juicio Moral) \\
- Inventario de Ideologías Educativas de \\
O'Neill. \\
-Cuestionario multifactorial sobre \\
liderazgo educacional. \\
-Rúbrica de evaluación del nivel de \\
implementación de PROVEE. \\
- Indicador de logro académico \\
(ENLACE)
\end{tabular}

Se aplicaron un total de 12,156 encuestas tanto a padres de familia, alumnos como a maestros de las escuelas, según los rasgos de la selección de la muestra. La siguiente tabla da cuenta de las encuestas aplicadas en pre-test como en pos-test, organizados por agente y tipo de muestra:

Cuadro 3. Encuestas de satisfacción de Clima Moral

\begin{tabular}{|l|c|c|c|c|c|c|}
\hline \multirow{2}{*}{} & \multicolumn{2}{|c|}{ Número encuestas de satisfacción de Clima Moral ${ }^{1}$ aplicadas } \\
\cline { 2 - 7 } & \multicolumn{2}{|c|}{ Maestros } & \multicolumn{2}{c|}{ Padres } & \multicolumn{2}{c|}{ Alumnos } \\
\cline { 2 - 7 } & Pres-test & Post-test & Pres-test & Post-test & Pres-test & Post-test \\
\hline Muestra A.1 & 311 & 377 & 978 & 1678 & 1176 & 1117 \\
\hline Muestra A.2 & 117 & 163 & 356 & 320 & 116 & 170 \\
\hline Muestra B & 225 & 214 & 890 & 978 & 765 & 868 \\
\hline Muestra C & 85 & 77 & 326 & 356 & 224 & 269 \\
\hline Total & $\mathbf{7 3 8}$ & $\mathbf{8 3 1}$ & $\mathbf{2 5 5 0}$ & $\mathbf{3 3 3 2}$ & $\mathbf{2 2 8 1}$ & $\mathbf{2 4 2 4}$ \\
\hline
\end{tabular}

\section{Hallazgos}

En el siguiente cuadro se presentan los hallazgos agrupados por las variables e indicadores del clima moral: 
Cuadro 6. Hallazgos organizados por variables e indicadores del Clima Moral.

\begin{tabular}{|c|c|c|}
\hline Valores & Variables & Indicadores observables \\
\hline $\begin{array}{c}\text { Conocimiento de sí } \\
\text { (aprecio por la dignidad } \\
\text { propia) }\end{array}$ & $\begin{array}{c}\text { Desarrollo personal y } \\
\text { profesional }\end{array}$ & $\begin{array}{c}\text { Percepción sobre el desarrollo } \\
\text { personal y profesional de los } \\
\text { docentes y su impacto en el } \\
\text { proceso educativo. }\end{array}$ \\
\hline
\end{tabular}

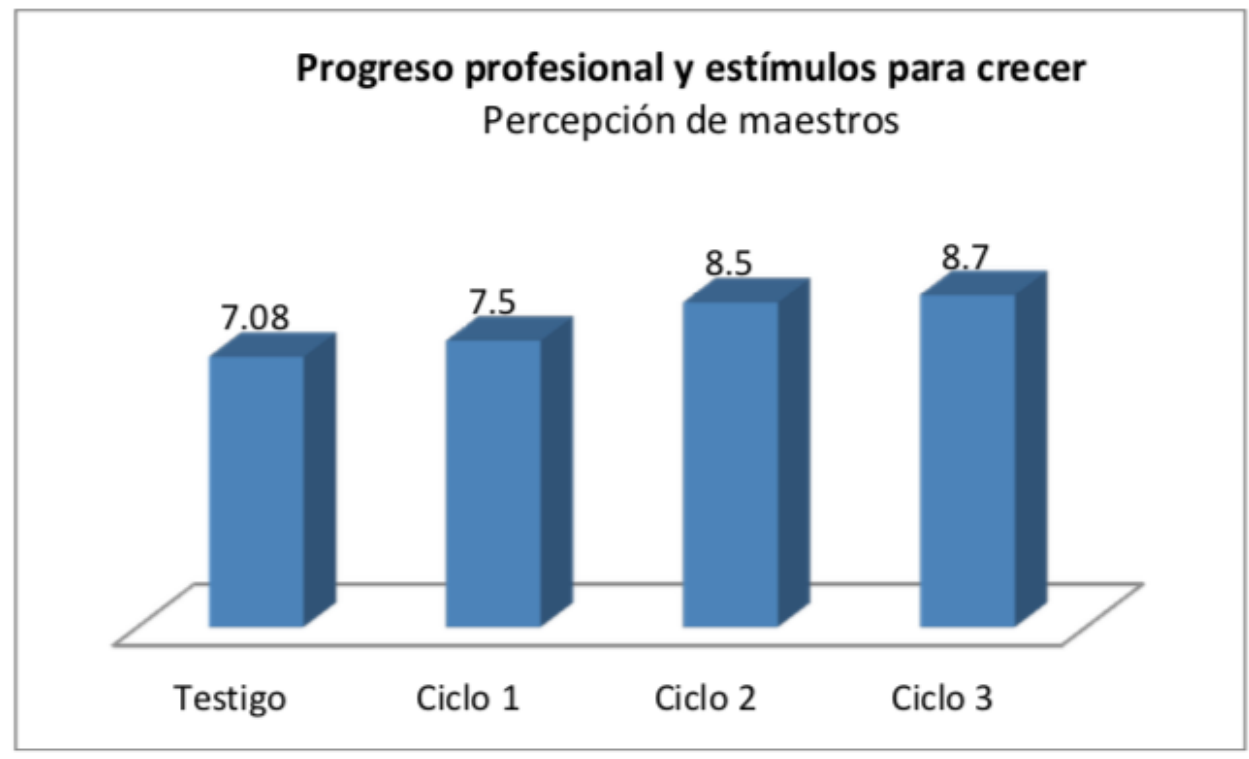

Cuadro 7.

\begin{tabular}{|c|c|c|}
\hline Valores & Variables & Indicadores observables \\
\hline & Procesos de vinculación al & $\begin{array}{c}\text { Procesos de comunicación e } \\
\text { información al interior del } \\
\text { colectivo escolar. }\end{array}$ \\
\cline { 3 - 3 } Solidaridad/empatía & $\begin{array}{c}\text { interior de la Escuela y } \\
\text { Escuela-Familia }\end{array}$ & $\begin{array}{c}\text { Comunicación y relación de la } \\
\text { escuela con los padres. }\end{array}$ \\
\hline
\end{tabular}

Fortaleciendo el Clima Moral en escuelas de educación básica en Ciudad Juárez 

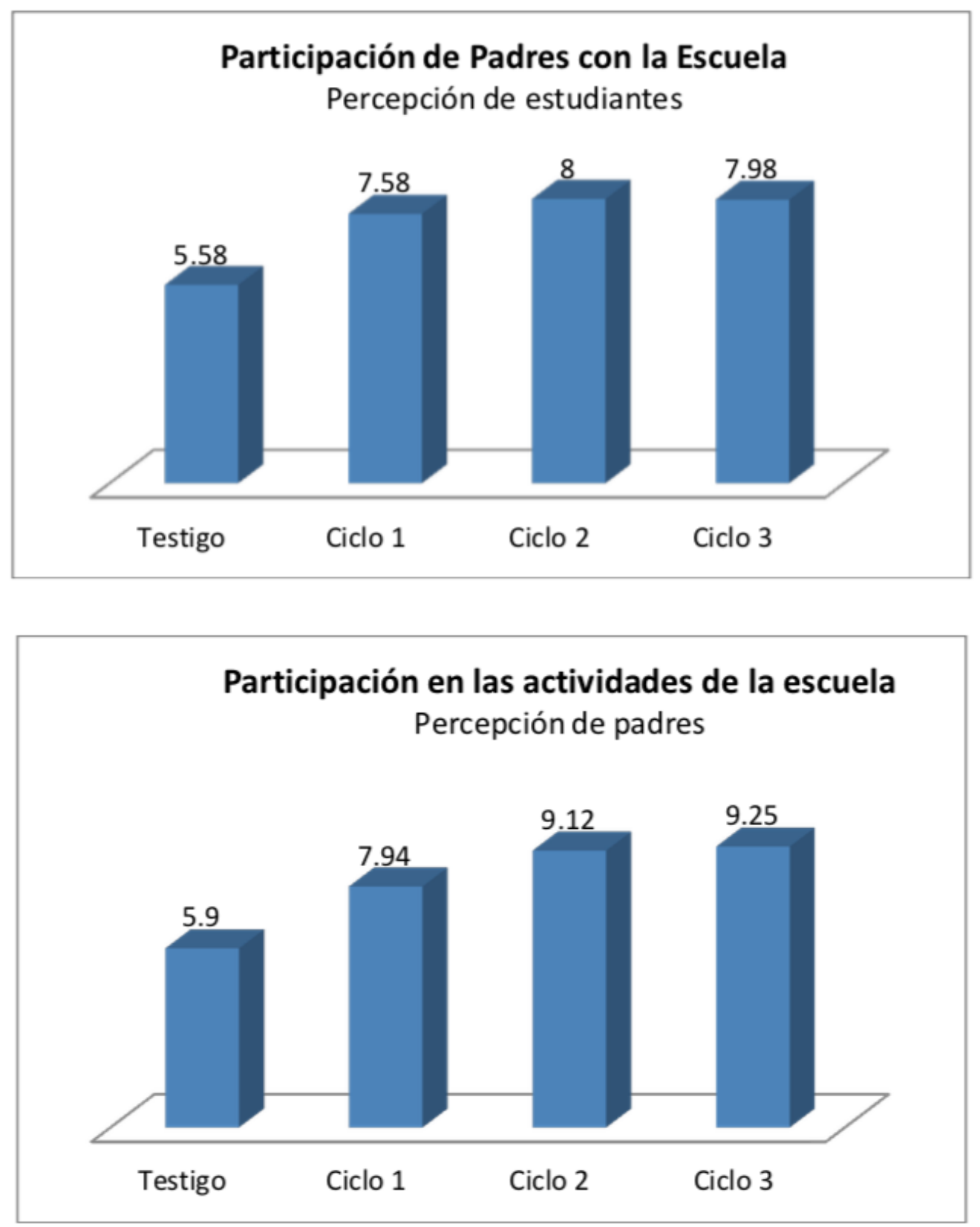

Cuadro 8.

\begin{tabular}{|c|c|c|}
\hline Valores & Variables & Indicadores observables \\
\hline $\begin{array}{c}\text { Sentido de justicia y } \\
\text { equidad. }\end{array}$ & Desarrollo de juicio moral & $\begin{array}{c}\text { Evaluación del nivel de } \\
\text { desarrollo del juicio moral. }\end{array}$ \\
\cline { 2 - 3 } & Ejercicio del liderazgo. & Diagnóstico de liderazgo \\
\hline
\end{tabular}


Cuadro 9.

\begin{tabular}{|c|l|c|}
\hline Valores & \multicolumn{1}{|c|}{ Variables } & Indicadores observables \\
\hline \multirow{4}{*}{$\begin{array}{c}\text { Convivencia social } \\
\text { armónica }\end{array}$} & $\begin{array}{l}\text { Calidad Relaciones } \\
\text { Interpersonales }\end{array}$ & $\begin{array}{c}\text { Relaciones interpersonales entre } \\
\text { los maestros. }\end{array}$ \\
\cline { 2 - 3 } & $\begin{array}{l}\text { Calidad en las relaciones de } \\
\text { trabajo y su orientación al } \\
\text { cumplimiento de los objetivos } \\
\text { educativos. }\end{array}$ & $\begin{array}{c}\text { Percepción sobre la calidad en } \\
\text { las relaciones de trabajo } \\
\text { padres. }\end{array}$ \\
\cline { 2 - 3 } & $\begin{array}{c}\text { Ideología educativa de los } \\
\text { docentes }\end{array}$ & $\begin{array}{c}\text { Diagnóstico de las ideologías } \\
\text { educativas de los docentes. }\end{array}$ \\
\hline
\end{tabular}
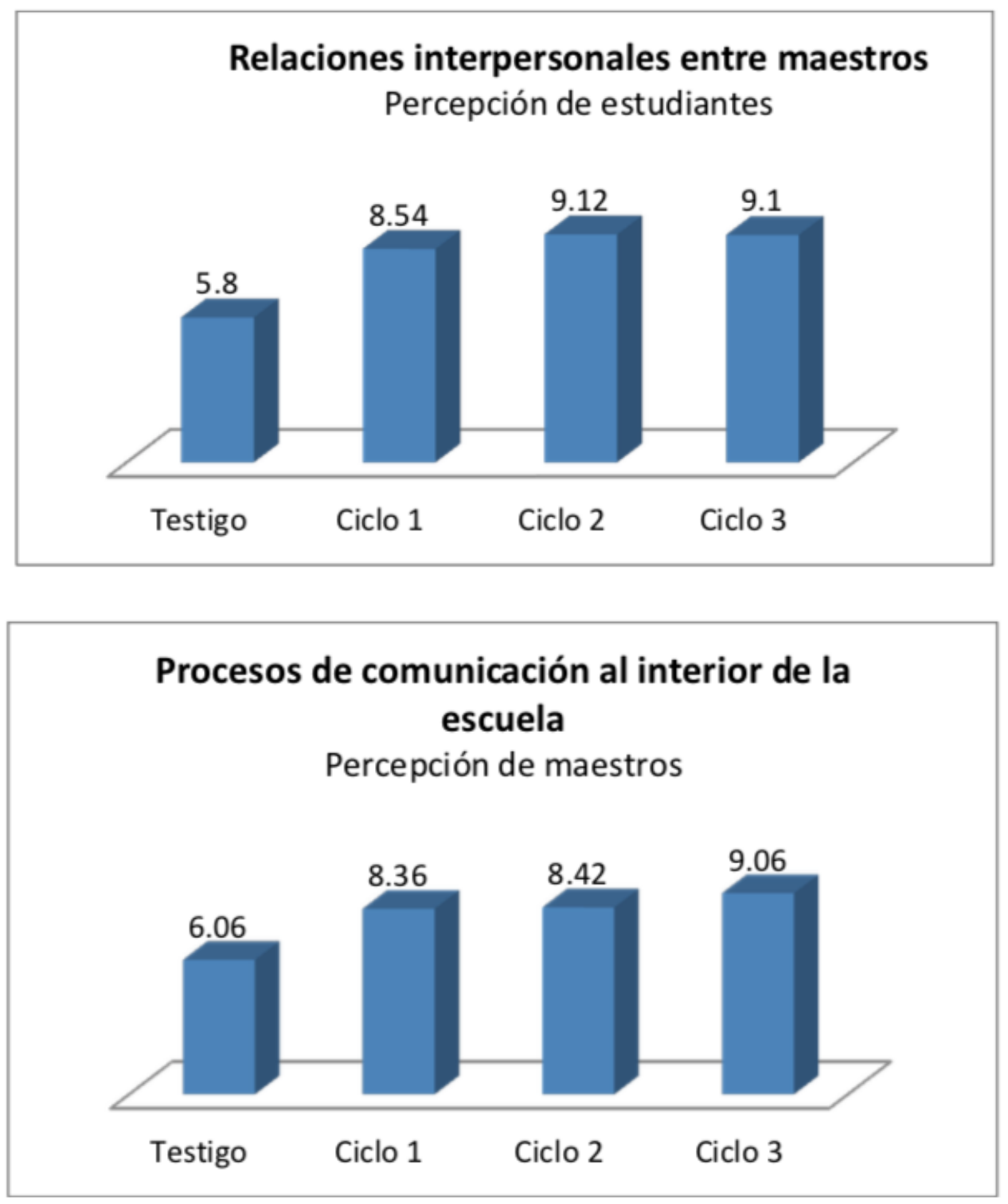

Fortaleciendo el Clima Moral en escuelas de educación básica en Ciudad Juárez 


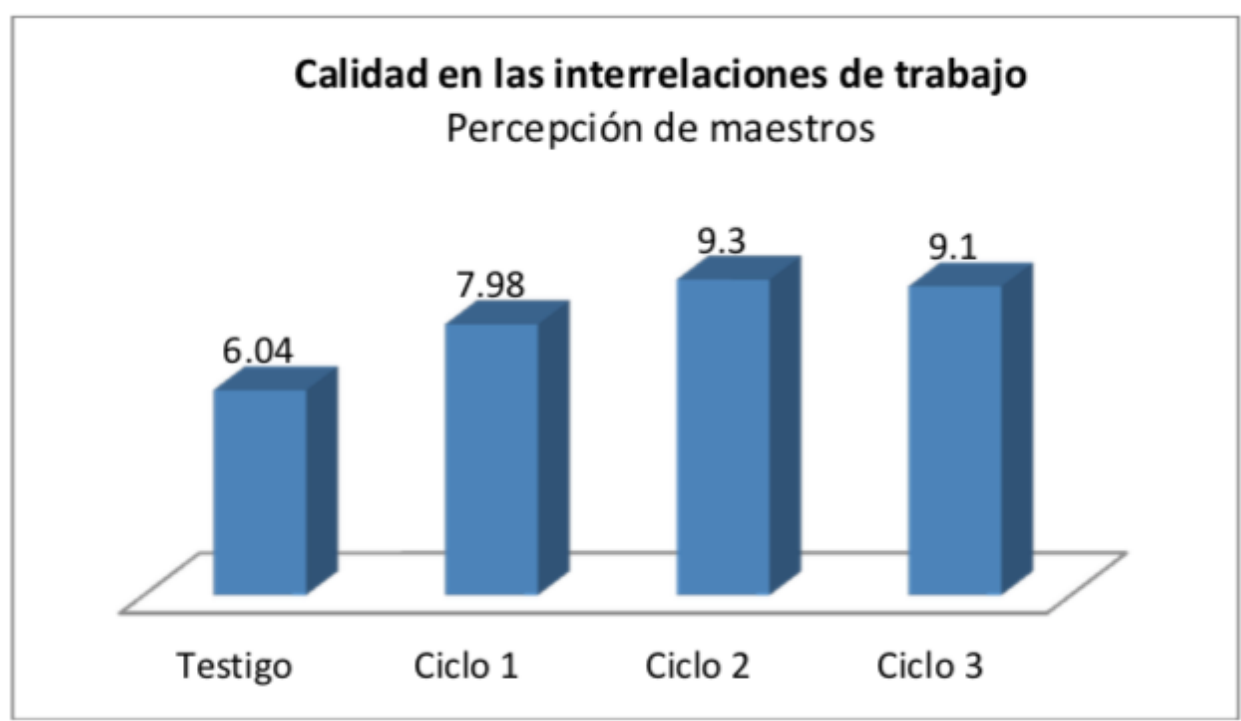

Cuadro 10.

\begin{tabular}{|c|l|l|}
\hline Valores & \multicolumn{1}{|c|}{ Variables } & \multicolumn{1}{|c|}{ Indicadores observables } \\
\hline & & $\begin{array}{l}\text { Establecimiento y } \\
\text { cumplimiento de objetivos en } \\
\text { el marco del proyecto escolar. }\end{array}$ \\
\cline { 3 - 4 } $\begin{array}{c}\text { Responsabilidad en la } \\
\text { asunción de tareas }\end{array}$ & $\begin{array}{l}\text { Cumplimiento de objetivos } \\
\text { educativos }\end{array}$ & $\begin{array}{l}\text { Conocimiento y apego del } \\
\text { colectivo escolar a la misión, } \\
\text { visión y valores que la escuela } \\
\text { busca a través de su proyecto } \\
\text { escolar. }\end{array}$ \\
\hline
\end{tabular}

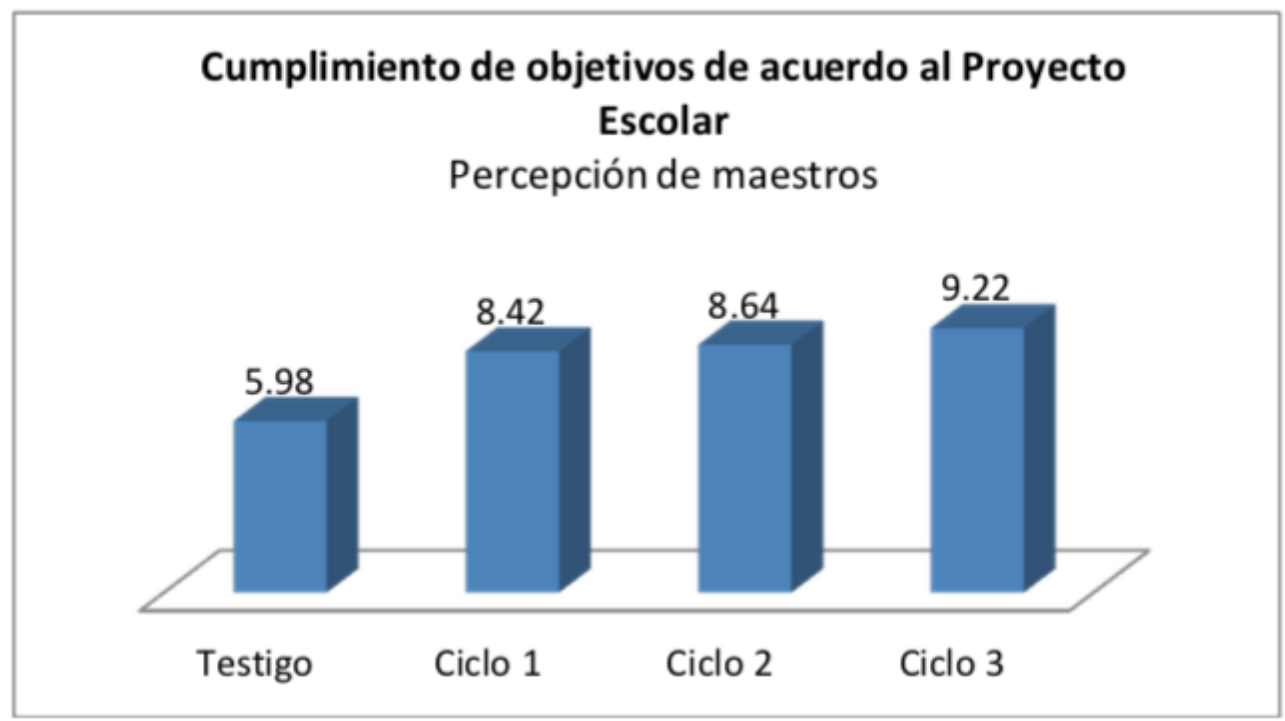




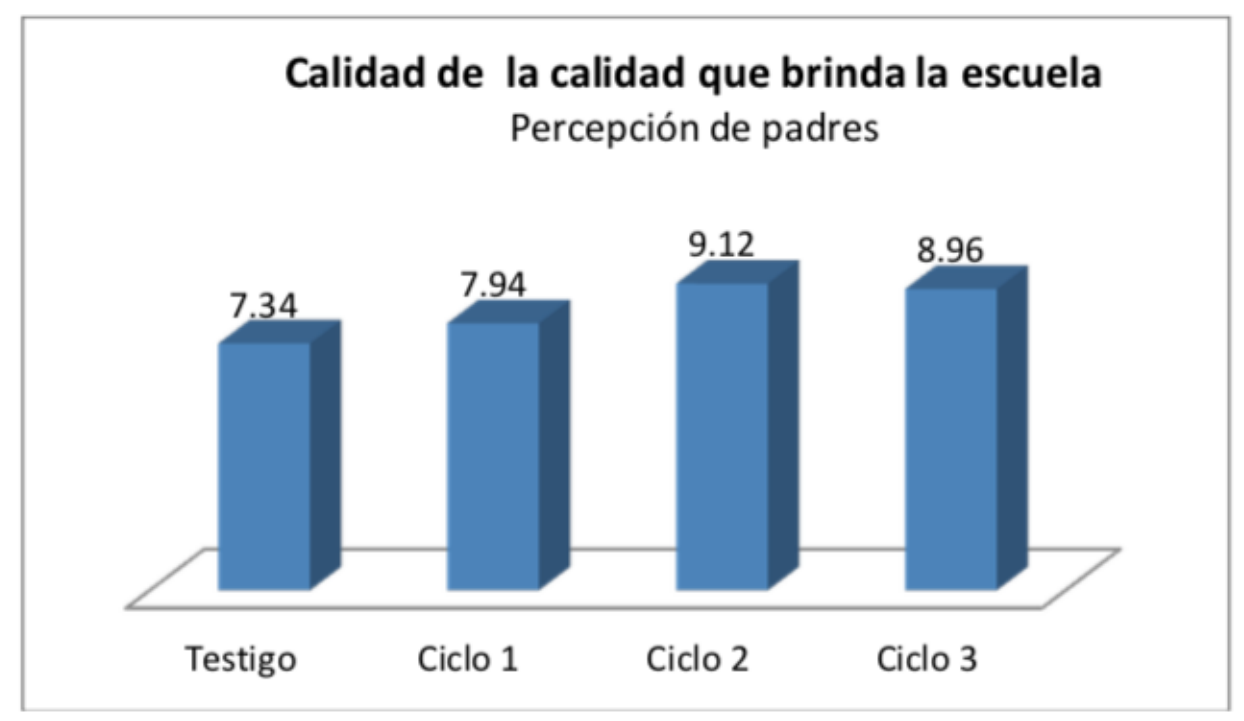

Cuadro 11.

\begin{tabular}{|c|l|l|}
\hline Valores & \multicolumn{1}{|c|}{ Variables } & \multicolumn{1}{|c|}{ Indicadores observables } \\
\hline \multirow{2}{*}{$\begin{array}{c}\text { Participación social } \\
\text { activa. }\end{array}$} & $\begin{array}{l}\text { Participación de los padres en } \\
\text { la tarea educativa }\end{array}$ & $\begin{array}{l}\text { Percepción sobre la relación de } \\
\text { los padres con la escuela. }\end{array}$ \\
\cline { 3 - 3 } & $\begin{array}{l}\text { Porcentaje de participación de } \\
\text { los padres en las actividades de } \\
\text { la escuela }\end{array}$ \\
\hline & $\begin{array}{l}\text { Participación en el proceso de } \\
\text { enseñanza-aprendizaje. }\end{array}$ \\
\cline { 2 - 3 } & $\begin{array}{c}\text { Proyección del trabajo de la } \\
\text { escuela en la comunidad } \\
\text { inmediata }\end{array}$ & $\begin{array}{l}\text { Percepción sobre la calidad de } \\
\text { la educación que imparte la } \\
\text { escuela. }\end{array}$ \\
\hline
\end{tabular}




\section{Conclusiones}

Finalmente se presentan las conclusiones generales de la investigación, organizadas por objetivos específicos:

Cuadro12. Conclusiones particulares y general

\begin{tabular}{|c|c|c|}
\hline & Objetivo(s) & Conclusión \\
\hline \multirow{3}{*}{ : } & $\begin{array}{l}\text { 1. Determinar el nivel de satisfacción del } \\
\text { clima moral en escuelas que desarrollan el } \\
\text { PROVEE en un ler ciclo de mejora } \\
\text { continua. (Fases I a la V). } \\
\text { ¿En qué medida ha mejorado el clima moral } \\
\text { en escuelas que desarrollan el PROVEE en } \\
\text { un ler ciclo de mejora continua (Fase V)?, } \\
\text { ¿existe diferencia entre las escuelas } \\
\text { ubicadas en la Fase V y la Fase I? }\end{array}$ & $\begin{array}{l}\text { En términos generales, el clima moral de } \\
\text { las escuelas que implementan el PROVEE } \\
\text { mejora sustancialmente entre la Fase III } \\
\text { (diagnóstico) y al término de la fase de } \\
\text { implementación (Fase V) que lo hace } \\
\text { establece la incidencia de la propuesta del } \\
\text { PEV. } \\
\text { Existe una diferencia significativa entre los } \\
\text { resultados cuantitativos obtenidos entre } \\
\text { escuelas de Fase I y escuelas de Fase V, lo } \\
\text { que permite establecer que los procesos } \\
\text { para la mejora del clima moral de la escuela } \\
\text { deben ser desarrollados de manera } \\
\text { consciente y a través de acciones } \\
\text { especificas, aspectos que considera la } \\
\text { propuesta del PEV a través del PROVEE. }\end{array}$ \\
\hline & $\begin{array}{l}\text { 2. Determinar el nivel de satisfacción del } \\
\text { clima moral en escuelas que desarrollan el } \\
\text { PROVEE en un 2do ciclo de mejora } \\
\text { continua (Fases V). } \\
\text { ¿En qué medida ha mejorado el clima moral } \\
\text { en escuelas que desarrollan el PROVEE en } \\
\text { un 2do. ciclo de mejora continua (Fase V)? }\end{array}$ & $\begin{array}{l}\text { A partir del segundo ciclo de } \\
\text { implementación del PROVEE los } \\
\text { resultados cuantitativos se estabilizan en la } \\
\text { mejora, pero la diferencia numérica } \\
\text { (medias) de los datos cuantitativos } \\
\text { (encuestas) es muy poco. Los datos } \\
\text { cualitativos levantados establecen una } \\
\text { incidencia del PROVEE en la construcción } \\
\text { del Clima Moral de la Escuela (como se } \\
\text { puede constatar en los indicadores } \\
\text { particulares). } \\
\text { Un aspecto que puede considerar es } \\
\text { reestructurar el instrumento (encuesta) a fin } \\
\text { de hacerlo más exhaustivo. }\end{array}$ \\
\hline & $\begin{array}{l}\text { 3. Determinar el nivel de satisfacción del } \\
\text { clima moral en escuelas que desarrollan el } \\
\text { PROVEE en un 3er. ciclo de mejora } \\
\text { continua (Fases V). }\end{array}$ & $\begin{array}{l}\text { El nivel del clima moral entre segundo y } \\
\text { tercer ciclo de implementación del } \\
\text { PROVEE se establece como sostenido en } \\
\text { un nivel entre satisfactorio-muy }\end{array}$ \\
\hline
\end{tabular}




\begin{tabular}{|c|c|c|}
\hline & $\begin{array}{l}\text { ¿En qué medida ha mejorado el clima moral } \\
\text { en escuelas que desarrollan el PROVEE en } \\
\text { un 3er ciclo de mejora continua (Fase V)? }\end{array}$ & $\begin{array}{l}\text { satisfactorio. Los datos cualitativos indican } \\
\text { una mejora sostenida aún y contra factores } \\
\text { de la dinámica del sistema: rotación de } \\
\text { personal y cambio de director, por } \\
\text { mencionar dos. } \\
\text { Se reitera la consideración para } \\
\text { reestructurar el instrumento (encuesta) a fin } \\
\text { de hacerlo más exhaustivo a fin de que } \\
\text { permita establecer, de existir, diferencias en } \\
\text { indicadores a través de los instrumentos } \\
\text { cuantitativos. }\end{array}$ \\
\hline 产 & $\begin{array}{l}\text { ¿En qué medida el proyecto de valores } \\
\text { éticos (PROVEE) que desarrolla el } \\
\text { Programa Educación en Valores en } \\
\text { escuelas del nivel básico en Cd Juárez, } \\
\text { Chihuahua contribuye a Mejorar el } \\
\text { clima moral de la institución mediante el } \\
\text { diseño y/o implementación de un } \\
\text { Proyecto de Valores Éticos (PROVEE), } \\
\text { y/o con apoyo en el desarrollo de talleres } \\
\text { de desarrollo humano a los integrantes } \\
\text { de los colectivos escolares, y de esta } \\
\text { manera permitir que a largo plazo se } \\
\text { impacte en la calidad educativa? }\end{array}$ & $\begin{array}{l}\text { El proyecto de valores éticos (PROVEE) } \\
\text { que desarrolla el Programa Educación en } \\
\text { Valores en escuelas del nivel básico en Cd } \\
\text { Juárez, Chihuahua impacta en la mejora del } \\
\text { clima moral en las escuela. Esta incidencia } \\
\text { se da a través el diseño y/o implementación } \\
\text { de un Proyecto de Valores Éticos } \\
\text { (PROVEE), en la medida en que avanzan } \\
\text { en los ciclos de implementación la } \\
\text { satisfacción de los agentes (maestros, } \\
\text { padres de familia y alumnos) es sostenida. } \\
\text { Los talleres de desarrollo humano } \\
\text { (Desarrollo Persona, Relaciones } \\
\text { Interpersonales y Liderazgo Moral) son un } \\
\text { factor que apoya las solución de problemas } \\
\text { de indole formativo, primero con el } \\
\text { colectivo de maestros y luego con los } \\
\text { padres de familia, se visualiza la necesidad } \\
\text { de establecer acciones que impacten de } \\
\text { manera directa en los estudiantes. } \\
\text { En el indicador de ENLACE de las escuelas } \\
\text { evaluadas no puede encontrarse un patrón } \\
\text { común de la manera que estas escuelas } \\
\text { trabajan para mejorar su logro educativo. }\end{array}$ \\
\hline
\end{tabular}

\section{Agradecimientos}

Agradecimiento especial al Programa Educación en Valores A.C (PEV) de Ciudad Juárez, y a su directora ejecutiva Lic. Juanamary R. de Orozco, por todas las facilidades brindadas para la realización de la investigación que soporta este artículo.

Fundación del Empresariado Chihuahuense- Juárez (FECHAC) y Fundación Rosario Campos de Fernández. Mtro. Guillermo Narro Garza, Director General de Educación en la Zona Norte.

\section{Referencias}

Anaya, R. (2012). Educación democrática y formación de ciudadanía. Razonamiento moral e ideología educativa en instituciones formadoras y actualizadoras de docentes en el estado de Chihuahua. Tesis de Doctorado en espera de defensa. Juárez, Chih. Universidad Autónoma de Ciudad Juárez.

Arroyo, I. (1998) Filosofía de la educación en Chihuahua. Edit. Instituto Chihuahuense de la Cultura. Chihuahua, Chih.

Cortina, A. (1994) 10 palabras clave en ética. Navarra: Editorial Verbo 
Divino. Cortina, Adela (1997) Ciudadanos del mundo: hacia una teoría de la ciudadanía.

Madrid:Alianza Editorial. Escamez, J., Perez-Delgado, E., Domingo, A., Escrivà, V; Pérez, C. (1998) Educar en la autonomía moral. Valencia: Generalitat Valenciana.

SEP: Evaluación Nacional del Logro Académico en Centros Escolares. Consultado, en http://www.enlace.sep.gob.mx/no ticias/noticia_2/

Ferman, O. I y Junco, R. I. (2008). Modelo Educativo del Programa Educación en Valores. Chihuahua, Chih.: Talleres Gráficos de Gobierno del Estado.

Garma, A.M.; Elexpuru, I. (1999) El autoconcepto en el aula. Recursos para el profesorado. Barcelona: Edebé.

Giroux, S. y Tremblay, G. (2002). Metodología de las ciencias humanas. Distrito Federal. México: Fondo de Cultura Económica.

Hansen, D. (2002) Explorando el corazón moral de la enseñanza. Barcelona: Idea Books. Hargreaves, Andy (1996) Profesorado, cultura $y$ postmodernidad: cambian los tiempos, cambia el profesorado. Madrid: Morata. Hernández, R., Fernández-Collado, C. y Baptista, P. (2006). Metodología de la Investigación. Distrito Federal. México: Mc Graw Hill.

Hersh, R. Paolito, D. Reimer, J. (1988). El crecimiento moral de Piaget a Kohlberg. Madrid: Ed. Narcea, S.A.

Kerlinger, Fred \& Lee, Howard. (2001). Investigación del comportamiento: Métodos de investigación en las Ciencias Sociales. Cuarta edición. Distrito Federal, México: Mc Graw
Hill.

Kohlberg, L. (1992) Psicología del desarrollo moral. Bilbao: Deslcée de Brouwer. Martínez, Miquel (2000) El contrato moral del profesorado. Bilbao, Desclée De Brouwer.

Martínez, Miquel; Bujons, Carlota (coord.) (2001) Un lugar llamado escuela: en la sociedad de la información y de la diversidad. Barcelona, Ariel.

Martínez, Miquel; Hoyos, Guillermo (coord.) (2004) ¿Qué significa educar en valores hoy? Barcelona: Octaedro-Organización de Estados Iberoamericanos.

Martínez, M.; Tey, A. Campo, A. (2006) «La educación en valores y el aprendizaje ético» en Por preguntar que no quede. Madrid: Ministerio de Educación y Ciencia.

Novak, J; Gowin, B. (1988) Aprendiendo a aprender. Barcelona: Martínez Roca.

Loera, A. (2005). Filosofía Educativa de los Maestros de las escuelas del Programa Escuelas de Calidad. En Reportes Nacionales fase 3, Evaluación Cualitativa del Programa Escuelas de Calidad. México, Distrito Federal: SEP.

O’Neill William F. (1981) "Educational ideologies: contemporary expressions of educational philosophy". Santa Mónica, Cal. USA: Edit. Goodyear Publisching Co.

Piaget, J. (1984) El criterio moral en el niño. Barcelona: Martínez Roca. Pascual, R., Villa, A., y Auzmendi, E. (1993). El liderazgo transformacional en los centros docentes. Bilbao: Ediciones Mensajero.

Reimer, Paolito y Hersch, (1983). Promoting moral grow from Piaget to Kohlberg. 2a ed. Prospect Heights, Waveland. En Schmelkes, 
S. (2004).

Schmelkes, S. (2004). La formación de valores en la educación básica. México: SEP.

Zvokey, D. (1990). Objectivity and moral judgment: Towards agreement on a moral education theory. Journal of Moral Education, Vol. 19. núm. I, enero. En Schmelkes, S. (2004). 
RECIE. Revista Electrónica Científica de Investigación Educativa

Vol. 1, núm. 1, enero-diciembre 2012, pp. 261-275.

Ferman Ávila, O.I. 